\title{
Spreading Frost Under the Microscope
}

\section{A new imaging technique reveals the effects of humidity on the spread of frost across a micropatterned surface.}

\section{By David Ehrenstein}

W ith October starting, those living in northern latitudes will soon have the chance to admire the dazzling ice patterns on frost-covered windows. To study frosting, a research team has combined a new frost imaging technique with a surface made of microscopic pillars. Their experiments reveal the various modes of frost spread across a surface with a new level of detail [1]. They found that at an intermediate level of humidity, frost spreads in fractal patterns.

Doris Vollmer of the Max Planck Institute for Polymer Research in Germany, Lou Kondic of the New Jersey Institute of Technology, and their colleagues created a surface consisting of a square lattice of micropillars 10 micrometers high, where the pillar diameter and spacing were both 30 micrometers. This micropatterned surface can control the locations of the

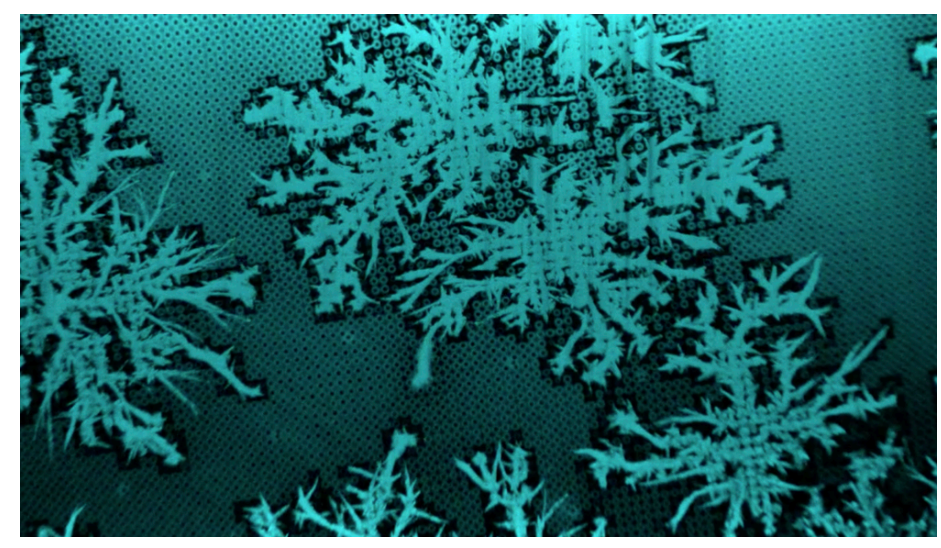

Frosty fractals. At an intermediate level of humidity, frost spreads across a micropatterned surface in a fractal pattern. (See videos below.)

Credit: L. Hauer et al. [1]

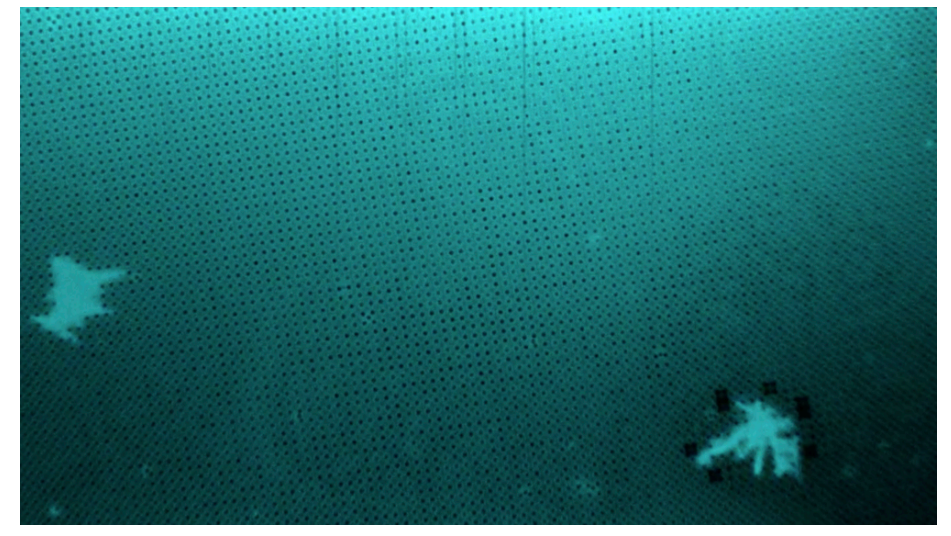

Under low humidity, only a small region of frost forms. Credit: L. Hauer et al. [1]

condensed droplets and allows detailed observations of the frosting process that are not possible with smooth and uniform surfaces. The team infused the surface with dyed silicone oil, which allowed them to use an imaging technique called laser-induced fluorescence microscopy to track ice formation. At $-30{ }^{\circ} \mathrm{C}$, the team demonstrated three different modes of frosting, at $14 \%, 24 \%$, and $34 \%$ relative humidity.

In each case, the process starts with supercooled liquid droplets forming on the pillars, after which some of these droplets freeze and become frost nucleation sites. At the lowest humidity level, the droplets evaporate quickly, so a frozen droplet cannot interact with others. Instead, it forms a small region of frost by extracting and freezing some water vapor from its surroundings.

At the highest humidity level, the droplet evaporation is 


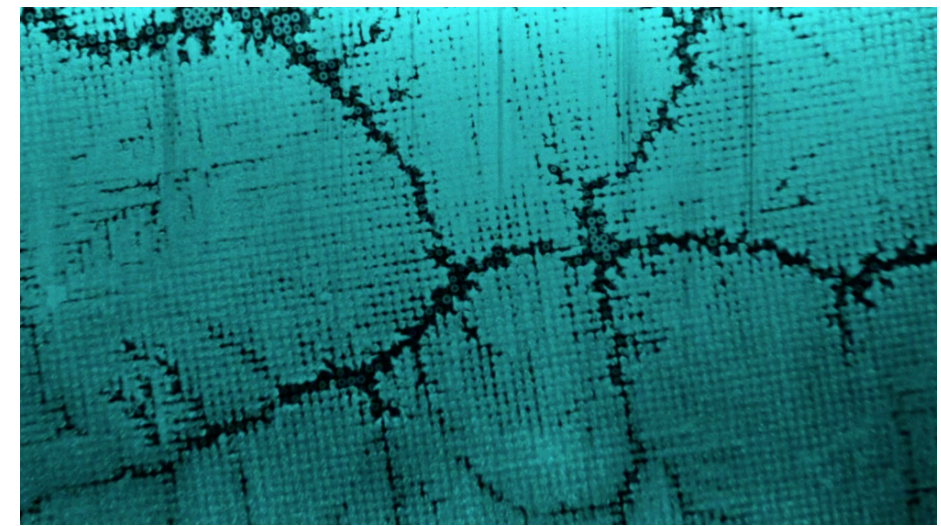

Under high humidity, the entire surface becomes frost covered. Credit: L. Hauer et al. [1]

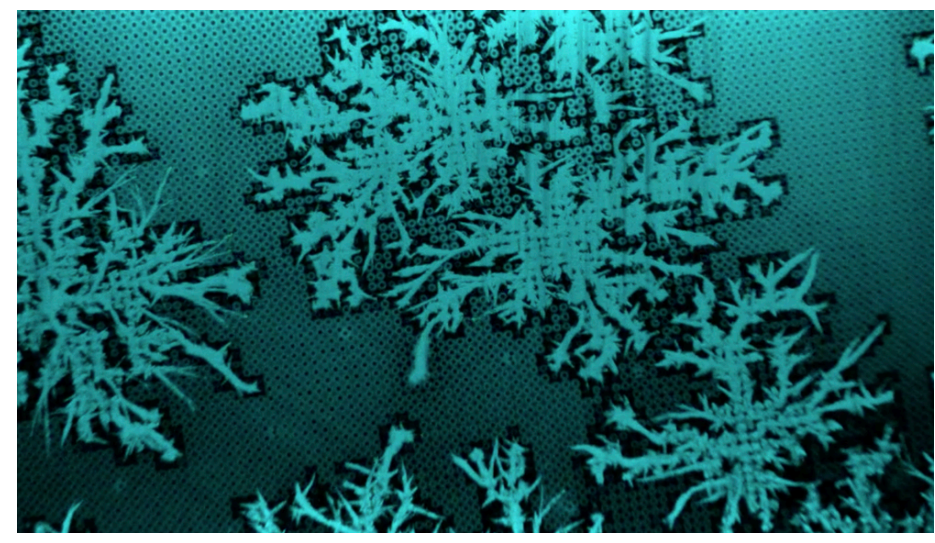

Under intermediate humidity, the frost produces a region with fractal geometry with "dry regions" as voids.

Credit: L. Hauer et al. [1] relatively slow. There is enough time for a frozen droplet to use the water evaporating from its neighbors to grow spikes that eventually touch the neighboring droplets, causing them to instantly freeze and form their own spikes. This chain reaction allows the frost to rapidly cover nearly every pillar.

At the intermediate humidity level, some droplets evaporate too quickly to be pulled into the chain reaction-leaving "dry regions"-while others do not. This mixed mode produces frost regions having fractal geometry, which had not been previously observed.

The researchers believe that their observations will lead to a better understanding of the details of the frosting process, which may in turn lead to technologies that protect power transmission lines, wind turbines, and other infrastructure from frost-induced damage.

David Ehrenstein is a Senior Editor for Physics.

\section{REFERENCES}

1. L. Hauer et al., "Frost spreading and pattern formation on microstructured surfaces," Phys. Rev. E 104, 044901 (2021). 